\title{
Liderazgo transformacional y gestión educativa en instituciones educativas nivel secundaria - Ugel Maynas - Loreto, 2018
}

\section{Transformational leadership and educational management in secondary level educational institutions - Ugel Maynas - Loreto, 2018}

\author{
Julia Antonieta Alarcón Aliaga ${ }^{1}$; Abelardo Rodolfo Campana Concha ${ }^{1}$
}

\begin{abstract}
RESUMEN
Objetivo: Determinar la relación entre liderazgo transformacional directivo y gestión educativa en instituciones educativas públicas secundaria, UGEL Maynas-Loreto, 2018. Materiales y Método: Es una investigación correlacional que aplicó un cuestionario en referencia a la variable Liderazgo transformacional y gestión educativa, con una muestra de 243 docentes, cuya información recaudada fue procesada en el programa SPSS. Resultados: Presenta una relación conjunta de 0,909 que establece una relación conjunta entre las variables estudiadas. Asimismo, se estableció una relación de 0,900; 0,937; 0,94 y 0,931 que hace referencia la relación entre liderazgo transformacional con la gestión institucional, administrativa, pedagógica y comunitaria, respectivamente. Conclusión: Se confirma la relación de las variables, se produce a nivel medio, consigue la misma intensidad de desarrollo en las dimensiones (institucional, administrativa, pedagógica y comunitaria).
\end{abstract}

Palabras clave: Liderazgo transformacional, gestión educativa, institucional, administrativa, pedagógica y comunitaria.

\begin{abstract}
Objective: To determine the relationship between managerial transformational leadership and educational management in secondary public educational institutions, UGEL Maynas-Loreto, 2018. Materials and Method: It is a correlational investigation that applied a questionnaire in reference to the variable $X$ and $Y$, with a sample of 84 teachers, whose information collected was processed in the SPSS program. Results: It presents a joint relationship of 0.909 that establishes a joint relationship between the variables studied. Likewise, a ratio of 0.900 was established; $0.937 ; 0.894$ and 0.931 which refer to the relationship between transformational leadership with institutional, administrative, pedagogical and community management, respectively. Conclusion: the relationship of the variables is confirmed, it occurs at a medium level, it achieves the same intensity of development in the dimensions (institutional, administrative, pedagogical and community).
\end{abstract}

Keywords: Transformational leadership, educational, institutional, administrative, pedagogical and community management.

\section{INTRODUCCIÓN}

Desde hace décadas, la educación en el Perú atraviesa por serias deficiencias, tal como indica el Proyecto Educativo Nacional al 2021:

'La gestión del aparato educativo se encuentra sumida en un marasmo de escasez de recursos, manejo ineficiente e inequitativo del presupuesto disponible, rigidez administrativa, burocratismo y, sobre todo, corrupción proliferante en todos los niveles (...)"(Minedu, 2007, p.32).

Esta problemática en la gestión, se refleja en los bajos niveles de logro en los estudiantes tal como se evidencia en el resultados obtenidos en la prueba internacional PISA en el 2012, (OCDE, 2014) medir la educación en educandos de 15 y 16 años, tanto: matemáticas, comprensión lectora y ciencias; sin embargo, entre 65 países, Perú quedó lugar último.

Por otro lado, el proyecto educativo regional de Loreto (PER) 2007-2021, presentado por el gobierno regional a través de la Gerencia Regional de Desarrollo Social, pone en evidencia un diagnóstico de la realidad de la educación en Loreto que corrobora lo planteado en el Proyecto Nacional y se refleja a través de logros de aprendizaje. Así, según la evaluación censal de estudiantes (ECE, 2015), cuyas pruebas estandarizadas fueron evaluados por Minedu, sólo el 4,5\% de los estudiantes alcanzaron nivel satisfactorio en Lectura y el
1,3\% alcanzaron nivel satisfactorio en Matemática, alertando problemas de aprendizaje a nivel nacional. De igual forma, en la ECE 2016 los estudiantes de secundaria aún siguen con bajos rendimientos, sólo $5,5 \%, 1.7 \%$ y $4,3 \%$ de los estudiantes lograron nivel satisfactorio en Historia, Geografía y Economía, Escritura y Matemática, respectivamente; en comparación con Tacna que obtuvo el primer puesto por obtener nivel satisfactorio de $26,6 \%, 30 \%$ y $28,9 \%$ en las mismas áreas. Estos resultados evidencian que los estudiantes de Loreto presentan deficiencias para lograr los aprendizajes esperados o en todo caso aún están en un nivel en proceso, por ende, no están preparados para afrontar los retos de aprendizaje del ciclo.

Estas cifras alarmantes, deben inducir a las instituciones educativas a reflexionar sobre la calidad en su gestión educativa. Según el Artículo 8 de la Ley General de Educación 29044, la calidad debe asegurar las condiciones adecuadas para una educación integral, pertinente, abierta, flexible y permanente. Calidad, que depende de la interacción de diversos factores como: infraestructura adecuada, implementación con recursos y herramientas educativas pertinentes, fomento de metodologías activas e innovadoras, planes curriculares idóneos, promoción de clima institucional adecuado y de armonía, trabajo docente entre otros. Aspectos, inmersos en la gestión educativa y que dependen, muchas veces, el liderazgo transformacional de los directores, aquel

Recibido:08/10/2020 - Aprobado:17/11/2020

${ }^{1}$ Estudiante de Doctorado de la Universidad Nacional Mayor de San Marcos

${ }^{2}$ Docente de Educación de la Universidad Nacional Mayor de San Marcos 
que motive a todo su personal a cumplir la misión y alcanzar la visión institucional; que sea capaz de apoyar al trabajo docente brindándole los recursos para un desempeño eficaz, que propicie un clima institucional sano, positivo y de crecimiento personal y fortalecimiento profesional; un promotor del cambio integral hacia la calidad educativa en beneficio de la comunidad escolar, local, regional y nacional.

Es decir, el director como líder de la institución educativa debe tener la capacidad de brindar un buen servicio en lo pedagógico, administrativo, institucional y comunitario con la satisfacción de aquellos que reciben este servicio bajo un liderazgo transformacional.

Siendo necesario, establecer la relación entre la capacidad de liderazgo transformacional directivo y gestión educativa. Así como la relación de los factores que están inmersos en estas variables que permitan orientar a las instituciones educativas hacia la mejora de la eficacia, tal como señala Page et al. (1990):

"[...] resultaría de gran utilidad fomentar investigaciones que estudiasen este ámbito, con objeto de subsanar los problemas de índole institucional, procedentes de la dirección y organización de centros, que pueden estar obstaculizando el rendimiento escolar de los alumnos" (p. 75)

Estudios anteriores como los de Thieme (2005) quien confirma que:

"Las dimensiones activas del liderazgo transformacional (Influencia idealizada, motivación por inspiración, estimulación intelectual/consideración individual, recompensa contingente y dirección por excepción-activo), se asocian positivamente con las variables de desempeño y las dimensiones pasivas del liderazgo se relacionan negativamente"

Sorados (2010), estableció que la influencia del liderazgo en la calidad de la gestión educativa, agregando que si los directores cuentan con buenas capacidades para liderar permitirán el incremento de la calidad del centro educativo, el cual será más evidente en el nivel pedagógico.

Calle (2008), quien en su interés por analizar el liderazgo transformacional y gestión institucional, encontró la necesidad que las instituciones requieren directores que inciten al cambio, siendo como objetivo tener una política de gestión, como innovador, tomen soluciones mirando el futuro.

\section{MATERIAL Y MÉTODOS:}

Es una investigación cuantitativa de diseño correlacional, que considera una población de 659 docentes, seleccionando una muestra de 243. A quienes se les aplicó la técnica de la encuesta y el cuestionario como instrumento. Se aplicó la fórmula de Pearson mediante el programa computacional SPSS.

\section{RESULTADOS}

Tabla 1

Frecuencias liderazgo transformacional

\begin{tabular}{lll}
\hline Niveles & Frecuencia & Porcentaje \\
\hline Bajo & 29 & 11.9 \\
Medio & 170 & 70.0 \\
Alto & 44 & 18.1 \\
TOTAL & 243 & 100.0 \\
& & \\
\hline
\end{tabular}

El $70 \%$ de docentes perciben como nivel medio, el $18,1 \%$ de los docentes opinan que el nivel es alto y el $11,9 \%$ de los docentes opinan que el nivel es bajo.

Tabla 2

Frecuencias de la gestión educativa

\begin{tabular}{lll} 
Niveles & Frecuencia & Porcentaje \\
\hline Bajo & 33 & 13,4 \\
Medio & 162 & 66,7 \\
Alto & 48 & 19,9 \\
TOTAL & 243 & 100,0 \\
& & \\
\hline
\end{tabular}

El $66,7 \%$ de los docentes opinan que la calidad de gestión educativa conducida por el director está en un nivel medio, el $19,9 \%$ opina que está en un nivel alto y el 13,4\% opina que está en un nivel bajo.

Prueba hipótesis general

HGA. El liderazgo transformacional directivo se vincula significativamente con la gestión educativa en instituciones educativas públicas de secundaria.

HGO. El liderazgo transformacional de los directores no se relaciona significativamente con la gestión educativa en instituciones educativas públicas de secundaria.

Tabla 3

Correlación de la hipótesis general

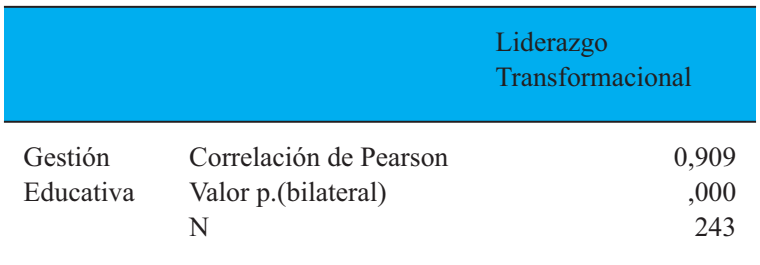

Se evidenció un Rho = 0,909 muy buena entre liderazgo transformacional y la gestión educativa.

\section{Contrastación hipótesis específica 1}

Ha. El liderazgo transformacional se relaciona significativamente con la calidad de la gestión institucional 
Tabla 4

Primera contrastación específica

\begin{tabular}{lll}
\hline & & $\begin{array}{l}\text { Liderazgo } \\
\text { Transformacional }\end{array}$ \\
\hline Gestión & Correlación de &, $900^{* *}$ \\
Institucional & $\begin{array}{l}\text { Pearson } \\
\text { Valor p.(bilateral) }\end{array}$ &, 000 \\
& N & 243 \\
\hline
\end{tabular}

Fuente: Alarcón (2018)

Se establece una correlación $\mathrm{RHO}=0,900$ entre liderazgo transformacional y gestión institucional.

Contrastación hipótesis específica 2

Ha. El liderazgo transformacional se relaciona significativamente con la calidad de la gestión administrativa

\section{Tabla 5}

Segunda contrastación específica

\begin{tabular}{lll}
\hline & & \multicolumn{1}{l}{$\begin{array}{l}\text { Liderazgo } \\
\text { Transformacional }\end{array}$} \\
\hline Gestión & $\begin{array}{l}\text { Correlación de } \\
\text { Administrativa }\end{array}$ &, $937^{* *}$ \\
& $\begin{array}{l}\text { Pearson } \\
\text { Valor p.(bilateral) }\end{array}$ &, 000 \\
& $\mathrm{~N}$ & 243 \\
\hline
\end{tabular}

Se halló un Rho = 0,937 entre el liderazgo transformacional y gestión administrativa.

\section{Contrastación hipótesis específica 3}

Ha. El liderazgo transformacional se relaciona significativamente con la calidad de la gestión pedagógica

Tabla 6

Tercera contrastación específica

\begin{tabular}{llr} 
& & $\begin{array}{l}\text { Liderazgo } \\
\text { Transformacional }\end{array}$ \\
\hline Gestión & Correlación de &, $894^{* *}$ \\
Pedagógica & Pearson & \\
& Valor p.(bilateral) &, 000 \\
& N & 243 \\
\hline
\end{tabular}

Se encontró Rho = 0,894 muy buena entre liderazgo transformacional y gestión pedagógica.

\section{Contrastación hipótesis específica 4}

Ha. El liderazgo transformacional se relaciona significativamente con la calidad de la gestión comunitaria
Tabla 7

Cuarta contrastación específica

\begin{tabular}{llr} 
& & \multicolumn{2}{l}{$\begin{array}{l}\text { Liderazgo } \\
\text { Transformacional }\end{array}$} \\
\hline Gestión & Correlación de &, $931^{* *}$ \\
Pedagógica & Pearson &, 022 \\
& Valor p.(bilateral) & 243
\end{tabular}

Asimismo, se determinó un Rho = 0,931 casi perfecta entre liderazgo transformacional y gestión comunitaria.

\section{DISCUSIÓN}

"La gestión actúa en los diferentes niveles de una organización para orientar en la perspectiva de los objetivos establecidos hacia la calidad y eficiencia. En ese sentido, la óptima gestión y formación integral de estudiantes, son dos de los principales objetivos que persiguen los directores transformacionales. Los directivos deben proponer soluciones y promover el cambio, motivar la justicia, estableciendo como individual y colectivo" (Pedraja, Rodríguez y Rodríguez, 2006; Murillo, 2006; Uribe, 2005, Mumford et al., 2000).

El análisis descriptivo permitió describir las variables de estudio y de acuerdo a las puntuaciones logradas, la capacidad de liderazgo del director, se ha ubicado en nivel medio $70 \%$, menciona, los directores no manejan el liderazgo óptimo; de igual forma, la puntuación de la variable gestión educativa se ubica en el nivel medio, dicho resultado indica que la gestión educativa no es óptima. Estos resultados encuentran similitud con los hallazgos de Párraga y Manco (2014) establecen, la asociación entre el liderazgo directivo y la labor del trabajador en el ámbito educativo, muestran que este tipo de liderazgo está en un nivel medio en un 59\%, y la variable desempeño del trabajador de servicio se ubica en un nivel medio en un $63 \%$. García (2015) investiga sobre liderazgo del personal directivo en la gestión de conflictos organizaciones, manifiesta que en el liderazgo transformacional, el porcentaje más elevado es en la escala de algunas veces con un $57,9 \%$ en la gestión de conflictos organizacionales.

Según los resultados obtenidos a través del análisis inferencial, se determinó la relación entre las variables, evidenciando que existe una relación significativa en un $95 \%$ (valor $p=, 000<0,05$ ) entre las variables en estudio. Esto quiere decir que el liderazgo transformacional del director se da cuando fortalece la cultura escolar, favorece el trabajo colaborativo, entabla la comunicación directa, comparte la autoridad y responsabilidad, presta estimulo intelectual a su personal, desarrolla una visión común para una conducción eficaz (Leithwood, 2004). El director debe prestar atención especial a la administración que implica el manejo de recursos materiales, técnicos, financieros dándoseles mayor importancia al recurso humano quien participa directamente en el proceso de mejoramiento. El director enfatizará la estimulación del esfuerzo docente (Mumford et al., 2000), porque la calidad educativa de las instituciones escolares "depende en gran parte de la gestión del director, su formación es considerada fundamental a la hora de tomar una decisión dirigida a lograr las metas de la institución" 
(Martin, Cammaroto, Neris, y Canelón, 2009, p.10).

Thieme (2005) quien estudió "la influencia del liderazgo en el desempeño escolar, a través de la eficiencia, esfuerzo extra y satisfacción con una escuela de la comuna de Arica - Chile, evidenció el desempeño escolar es significativamente mayor cuando los líderes son mejor evaluados, que cuando obtienen baja puntuación en la encuesta". Siendo la influencia del líder, factor determinante.

Otra investigación con similitud es la de Martínez (2007) respecto "al liderazgo transformacional en la gestión educativa de una institución educativa pública manifiesta que todos los docentes participantes identificaron tres de 10 características del liderazgo transformacional en el director: la influencia en el docente $(91,5 \%)$, la motivación en el docente $(93,5 \%)$ y la estimulación del docente $(96,1 \%)$. Un grupo de ellos le reconocieron otras características como su autoridad, poder de convencimiento y apoyo en el trabajo".

\section{CONCLUSIONES}

Se ha establecido que la capacidad de liderazgo transformacional a un nivel medio está relacionada con la misma intensidad con la gestión educativa, gestión institucional, gestión administrativa, gestión pedagógica y gestión comunitaria,

\section{AGRADECIMIENTO}

Alos docentes que formaron parte de esta investigación.

\section{REFERENCIAS BIBLIOGRÁFICAS}

Calle, C. (2008). Relación entre el liderazgo transformacional y la gestión institucional de los directores del nivel secundario de las Instituciones Educativas Públicas de la Región Callao. (Tesis de Licenciatura). Universidad Enrique Guzmán y Valle - La Cantuta, Lima, Perú

García, G. (2015). Liderazgo del personal directivo en la gestión de conflictos organizacionales, Institución Educativa "César Vallejo", Iquitos - 2015 (Tesis Magisterial). UNAP, Iquitos, Perú.

Leithwood, K. (2004). El liderazgo con éxito. El liderazgo educacional transformador en un mundo de políticos transaccionales. España: ICE Deusto.

Martin, F.; Cammaroto, A.; Neris, L. y Canelón, E. (2009). Liderazgo transformacional y gestión educativa en contextos descentralizados. Actualidades Investigativas en Educación, 9 (2), 1-27. Recuperado de https://www.redalyc.org/pdf/447/44713058008.pdf

Martínez, Y. (2007). El liderazgo transformacional en la gestión educativa de una institución educativa pública en el distrito de Santiago de Surco (Tesis Magisterial). PUCP, Lima, Perú.

Minedu (2007). Proyecto Educativo Nacional al 2021. $\mathrm{R}$ e c u p e r a d o d e http://www.minedu.gob.pe/Delnteres/xtras/PEN2021.pdf
Mumford, M. et al. (2000). Leadership skills: Conclusions and future directions. Leadership Quarterly, 11. $R$ e c u p e r a d o d e http://connection.ebscohost.com/c/articles/2987733/le adership-skills-conclusions-future-directions

Murillo, J (2006). Una dirección escolar para el cambio: del liderazgo transformacional al liderazgo distribuido. Revista Electrónica Iberoamericana sobre Calidad, Eficacia y Cambio en Educación, 4 (4), 11-24. $R$ e c u p e r a d o d e http://www.rinace.net/arts/vol4num4e/art2.pdf

Organización para la Cooperación y el Desarrollo Económico (2014). Resultados de PISA 2012 en Foco. $\mathrm{R}$ e c u p e r a d o d e https://www.oecd.org/pisa/keyfindings/PISA2012_Ove rview_ESP-FINAL.pdf

Page, M. et al. (1990). Hacia un modelo casual del rendimiento académico. España: CIDE

Párraga, A. y Bartolo, A. (2014). Liderazgo transformacional del director y el desempeño del trabajador del personal de las instituciones educativas públicas de la Red, Ate, 2014. (Tesis Magisterial). Universidad Cesar Vallejo, Lima, Perú.

Pedraja, L.; Rodríguez, E.; Rodríguez, J. (2006). Sociedad del conocimiento y dirección estratégica: una propuesta integradora. Interciencia, 31 (8), 570-576. Recuperado de https://www.redalyc.org/pdf/339/33911904.pdf

Sorados, P. M. M. (2010). Influencia del liderazgo en la calidad de la gestión educativa. Tesis para optar el grado de Magíster en Educación con Mención en Gestión de la Educación. Facultad de Educación. Universidad nacional Mayor de San Marcos, Lima.

Thieme, C. (2005). Liderazgo y eficacia en la educación primaria. El caso de Chile (Tesis doctoral). UAB, E s p a ñ . R e c u p e r a d o d e https://www.tdx.cat/bitstream/handle/10803/3958/cptj1 de1.pdf

Uribe, M. (2005). El liderazgo docente en la construcción de la cultura escolar de calidad: un desafío de orden superior. Recuperado de https://abacoenred.com/wpcontent/uploads/2015/10/El-liderazgo-docente-enconstrucci\%C3\%B3n-de-cultura-escolar-de-calidadUribe-M.-2005-pdf.pdf 